\title{
2-picolylamine derivatization for high sensitivity detection of abscisic acid in apicomplexan blood-infecting parasites
}

Ernest Moles $^{\mathrm{a}, \mathrm{b}, \mathrm{c}, *}$, Josep Marcos ${ }^{\mathrm{d}, \mathrm{e}}$, Santiago Imperial ${ }^{\mathrm{c}, \mathrm{f}}$, Oscar J. Pozo ${ }^{\mathrm{d}}$, Xavier Fernàndez-Busquets ${ }^{\mathrm{a}, \mathrm{b}, \mathrm{c}, *}$

a Nanomalaria Group, Institute for Bioengineering of Catalonia (IBEC), Baldiri Reixac 10-12, ES-08028 Barcelona, Spain

${ }^{b}$ Barcelona Institute for Global Health (ISGlobal, Hospital Clínic-Universitat de Barcelona), Rosselló 149-153, ES-08036 Barcelona, Spain

${ }^{c}$ Nanoscience and Nanotechnology Institute (IN2UB), University of Barcelona, Martí $i$ Franquès 1, ES-08028 Barcelona, Spain

${ }^{d}$ Bioanalysis Research Group, IMIM, Hospital del Mar, Doctor Aiguader 88, ES-08003 Barcelona, Spain

${ }^{\text {e }}$ Department of Experimental and Health Sciences, Universitat Pompeu Fabra, Doctor Aiguader 88, ES-08003 Barcelona, Spain

${ }^{f}$ Department of Biochemistry and Molecular Biomedicine, University of Barcelona, Avda. Diagonal 643, ES-08028 Barcelona, Spain

* Corresponding author at: Nanomalaria Group, ISGlobal, Rosselló 149-153, ES-08036 Barcelona, Spain.

E-mail address: ernest.moles@isglobal.org; xfernandez_busquets@ub.edu

\section{ABSTRACT}

We have developed a new liquid chromatography-electrospray ionization tandem mass spectrometry methodology based on 2-picolylamine derivatization and positive ion mode detection for abscisic acid (ABA) identification. The selected derivatization reaction leads to the formation of an amide derivative which contains a highly active pyridyl group. The enhanced ionization allows for a 700-fold increase over commonly monitored underivatized $A B A$, which in turn leads to excellent limits of detection and quantification values of 0.03 and $0.15 \mathrm{ng} \mathrm{mL}^{-1}$, respectively. This method has been validated in the highly complex matrix of a red blood cell extract. In spite of the high sensitivity achieved, ABA could not be detected in Plasmodium falciparum-infected red blood cells, suggesting that, if present, it will be found either in ultratrace amounts or as brief bursts at defined time points within the intraerythrocytic cycle and/or in the form of a biosynthetic analogue.

Keywords: Liquid chromatography-electrospray ionization tandem mass spectrometry; Abscisic acid; Picolylamine; Malaria; Plasmodium falciparum; Apicomplexa 


\section{Introduction}

Human-infecting intracellular parasites of the Apicomplexa phylum are responsible for millions of clinical cases resulting in thousands of deaths every year, mainly in developing tropical regions [1]. The Apicomplexa with higher clinical impact can be classified into three principal genera/diseases: Plasmodium/malaria, Babesia/babesiosis and Toxoplasma/toxoplasmosis. All these pathogens share a characteristic organelle, the apicoplast, a non-photosynthetic plastid which has several metabolic pathways absent in humans and therefore is considered an exceptional target for antiparasitic drug research [2]. Isoprenoid biosynthesis operating in plastidcontaining parasites has driven special attention because it is essential for the synthesis of numerous downstream metabolites that include ubiquinone, carotenoids and prenylation groups [3]. Carotenoid biosynthesis in the apicoplast has been described in Plasmodium falciparum [4] and in Toxoplasma gondii [5]. Considering the absence of photosynthetic activity in apicomplexan plastids, diverse alternative roles have been proposed for carotenoid derivatives in these parasites, including, among others [6-8], substrates for the synthesis of plant-like hormones that regulate essential cellular processes $[5,9,10]$. In this regard, abscisic acid (ABA, Fig. S1) has been proven to be endogenously produced by $T$. gondii through the $\mathrm{C}_{40}$-carotenoid biosynthetic pathway $\left(\mathrm{C}_{40}-\mathrm{CBP}\right)[5]$. ABA is necessary for Toxoplasma reinvasion, regulating the maturation and egress of parasites from the host infected cell in a complex calciumdependent signaling cascade [3]. The identification in $P$. falciparum of an host cell egress mechanism analogous to that present in $T$. gondii, together with the growth dependence of the parasite on intracellular oscillating calcium levels during most of its intraerythrocytic stage [11], are consistent with the possible existence of ABA or an ABA-like hormone in this pathogen. Accordingly, the herbicide fluridone (Fig. S1), which targets the phytoene desaturase enzyme in the early steps of the $\mathrm{C}_{40}-\mathrm{CBP}$, efficiently delayed $T$. gondii growth and prevented its multiplication while significantly reducing $A B A$ levels in the intracellular parasite [5]. Other compounds with an even stronger effect on ABA synthesis, such as the lipoxygenase inhibitor nordihydroguaiaretic acid (NDGA, Fig. S1), have been identified in plants [12] but to date have not been assayed against apicomplexan parasites.

Liquid chromatography-electrospray ionization tandem mass spectrometry (LCESI-MS/MS) is currently considered the technique of choice for the determination of $A B A$ and other phytohormones [13-17], providing excellent sensitivity, specificity and 
throughput, and permitting the analysis of non-volatile and thermally unstable compounds. Although the sensitivity of LC-ESI-MS/MS (in the range of a few $\mathrm{ng} \mathrm{mL}^{-1}$ ) allows the detection of endogenous levels of $A B A$ in plant material, significantly smaller concentrations of this hormone are expected to be produced by apicomplexan parasites due to the absence of most plant-like, ABA-regulated processes in these pathogens. This calls for the availability of high-sensitivity ABA detection protocols. Several chemical derivatization procedures have been developed in order to enhance the detection responses of carboxylic acid groups, such as the one present in $A B A$, in LC-ESI-MS/MS [18]. Picolylamine (PA), a derivatizing agent displaying a highly ESIactive pyridyl group that reacts with carboxylic acids in the presence of a condensation agent to form an amide derivative, has been employed for the analysis of fatty acids and other carboxyl-containing compounds [19].

In the present work we propose, for the first time, the use of PA for the derivatization of $A B A$ as the basis of an ultra-high sensitive approach for the detection of this compound in red blood cell-infecting apicomplexan parasites, this being in turn a more efficient protocol for ABA determination than the currently employed gas chromatography-MS assay, which requires the methylation of ABA [5]. In addition to the optimized detection parameters, the results obtained after the application of a full validation protocol for ABA detection in $P$. falciparum are presented.

\section{Materials and methods}

\subsection{Chemicals and reagents}

ABA and deuterated $A B A\left(\left[{ }^{2} H_{6}\right] A B A\right.$ or $A B A-d 6$; used as internal standard, ISTD), 2-PA, 2,2'-dipyridyl disulfide (DPDS), triphenylphosphine (TPP) and saponin were purchased from Sigma-Aldrich Corporation (St. Louis, MO, USA). Ethyl acetate, methanol (LC gradient grade), formic acid and ammonium formate (LC/MS grade) were obtained from Merck (Darmstadt, Germany). Ultrapure water was obtained from a MilliQ purification system (Millipore Ibérica, Barcelona, Spain).

\subsection{ABA purification from cell extracts and derivatization}

\subsubsection{Liquid-liquid extraction (LLE)}

An aliquot of $150 \mu \mathrm{L}$ of the lyophilized cell extract (Supplementary material) previously re-dissolved in $1 \mathrm{~mL}$ of acetonitrile:water $(8: 2 \mathrm{v} / \mathrm{v})$ was supplemented with 25 
$\mu \mathrm{L}$ of $1 \mathrm{ng} \mathrm{mL} \mathrm{m}^{-1} \mathrm{ABA}-\mathrm{d} 6$ and the mixture was evaporated under $\mathrm{N}_{2}$ stream at $40{ }^{\circ} \mathrm{C}$. After addition of $1 \mathrm{~mL}$ of a saturated $\mathrm{NaCl}$ solution and $1 \mathrm{~mL}$ of $1 \mathrm{M}$ acetic acid, the mixture was extracted with $6 \mathrm{~mL}$ of ethylacetate by shaking in a rocking mixer at 40 oscillations $\mathrm{min}^{-1}$ during $20 \mathrm{~min}$. After centrifugation (3000 $\mathrm{g}, 5 \mathrm{~min}$ ), the organic phase rich in protonated $A B A$ was separated and evaporated to dryness under a $\mathrm{N}_{2}$ stream coupled to a $40{ }^{\circ} \mathrm{C}$ water bath.

\subsubsection{Solid phase extraction (SPE)}

Alternatively to LLE, the lyophilized cell extracts re-dissolved in $1 \mathrm{~mL}$ of acetonitrile:water $(8: 2 \mathrm{v} / \mathrm{v})$ were processed by SPE. The mixture of re-dissolved extract and ABA-d6 was vortexed and passed through a Sep-Pak ${ }^{\circledR}$ Vac RC (500 mg) C18 cartridge (Waters Associates, Milford, MA, USA) previously conditioned with $\mathrm{MeOH}$ and washed with deionized water ( $2 \mathrm{~mL}$ of each). The column was rinsed with $2 \mathrm{~mL}$ deionized water and ABA was eluted with $2 \mathrm{~mL} \mathrm{MeOH}$. The methanolic extract was evaporated to dryness under a $\mathrm{N}_{2}$ stream in a $40{ }^{\circ} \mathrm{C}$ water bath.

\subsubsection{ABA derivatization with 2-PA}

The resulting dry pellet from either LLE or SPE was derivatized based on the procedure described by Higashi et al. [18]. Briefly, $10 \mu \mathrm{L}$ each of freshly prepared 10 $\mathrm{mM}$ TPP, $10 \mathrm{mM}$ DPDS, and $1 \mathrm{mg} \mathrm{mL}^{-1}$ 2-PA solutions in acetonitrile were successively added to the pellet. The mixture obtained was incubated at $60^{\circ} \mathrm{C}$ for 10 min, and after removal of the solvent by evaporation under a $\mathrm{N}_{2}$ stream at $40{ }^{\circ} \mathrm{C}$, the product was reconstituted with $150 \mu \mathrm{L}$ of deionized water:acetonitrile $(9: 1 \mathrm{v} / \mathrm{v})$ and 10 $\mu \mathrm{L}$ of the resulting solution were injected into the LC-MS/MS system.

\section{3. $L C-E S I-M S / M S$}

PA-derivatized ABA (ABA-PA) was quantified by LC-ESI-MS/MS with a selected reaction monitoring (SRM) method using a triple quadrupole (Quattro Premier XE) mass spectrometer equipped with an orthogonal Z-spray-electrospray ionization source (Waters Associates) interfaced to an Acquity UPLC system (Waters Associates) for the chromatographic separation. Nitrogen was employed as both drying and nebulising gas. The desolvation gas flow was set to approximately $1200 \mathrm{~L} \mathrm{~h}^{-1}$ and the cone gas flow to $50 \mathrm{~L} \mathrm{~h}^{-1}$. A cone voltage of $25 \mathrm{~V}$ and a capillary voltage of $3.0 \mathrm{kV}$ were used in positive ionization mode. The nitrogen desolvation temperature was set at $450{ }^{\circ} \mathrm{C}$ and the source temperature at $120^{\circ} \mathrm{C}$. The LC separation was performed using an Acquity 
UPLC BEH C18 column ( $100 \times 2.1 \mathrm{~mm}$ i.d., $1.8 \mu \mathrm{m}$, Waters Associates $)$ at a flow rate of $300 \mu \mathrm{L} \mathrm{min}^{-1}$. Water and methanol, both containing $0.01 \%$ formic acid and $1 \mathrm{mM}$ ammonium formate, were selected as mobile phase solvents. A gradient program was used where the percentage of organic solvent was linearly changed as follows: 0 min, 30\%; $0.5 \mathrm{~min}, 30 \%$; $5 \mathrm{~min}, 70 \%$; $5.5 \mathrm{~min}, 90 \%$; $6 \mathrm{~min}, 30 \%$; $7.5 \mathrm{~min}, 30 \%$. ABA-PA and ABA-d6-PA (PA-derivatized ISTD) were determined by SRM considering three transitions for each compound.

\subsection{Method validation for $A B A$ detection and quantification}

The samples selected for validation were processed through LLE plus PAderivatization (see Methods section 2.2) and finally analyzed by means of the LC-ESIMS/MS system described in Methods section 2.3. In each of three validation assays, precision and accuracy of the method were checked by running quality control samples (QCs) prepared by spiking lyophilized red blood cell (RBC) extracts with two different concentrations of ABA (0.15 and $\left.1.5 \mathrm{ng} \mathrm{mL}^{-1}\right)$. Calibration standards were analyzed and calibration curves were calculated by least-squares linear regression.

Six replicates were done for each level to evaluate intra-assay reproducibility and accuracy, which were expressed, respectively, as the relative standard deviation of the estimated concentrations obtained for the six QCs analyzed in one assay, and as the recovery value (\%) estimated for the QCs. Inter-assay reproducibility and accuracy were evaluated similarly from all replicates analyzed in three validation assays by two different operators $(n=12)$. Limits of detection (LOD) and quantification (LOQ) were defined, respectively, as the lowest concentration displaying a chromatogram signal/noise ratio $(\mathrm{S} / \mathrm{N})$ value of 3 , and as the concentration which gives a $S / N$ value of 10. ABA concentration for the determination of LOD and LOQ was calculated considering the lyophilized RBC extract resuspended in $1 \mathrm{~mL}$ acetonitrile:water (8:2 $\mathrm{v} / \mathrm{v}$ ), as described in Methods section 2.2, which results in approximately $1.2 \times 10^{10}$ RBCs $\mathrm{mL}^{-1}$.

\subsection{Evaluation of the RBC matrix effect}

Six blank samples corresponding to $150-\mu \mathrm{L}$ replicates of a re-dissolved RBC extract were processed by LLE and the resulting dry pellet was subsequently supplemented with $150 \mu \mathrm{L}$ of $5 \mathrm{ng} \mathrm{mL}^{-1} \mathrm{ABA}$ and $25 \mu \mathrm{L}$ of $1 \mathrm{ng} \mathrm{mL} \mathrm{m}^{-1} \mathrm{ABA}-\mathrm{d} 6$. The same amounts of $A B A$ and $A B A-d 6$ were added in parallel to three clean tubes. The 
derivatization reaction with PA was thereafter performed in all nine tubes. Matrix effect in both $A B A$ and ABA-d6 was calculated by means of the comparison between the responses of the RBC extract blank samples spiked after LLE and the solvent standards.

\section{Results}

\subsection{Optimization of the method for ABA detection}

The mass spectrometric parameters for PA-derivatized ABA (ABA-PA and ABAd6-PA ISTD) were optimized in order to achieve an increased signal (Table 1). ABA derivatization (Fig. 1A) proved to notably increase the MS/MS response towards this metabolite when compared to the underivatized species, an effect being illustrated by the chromatograms corresponding to the analysis of the same amounts of ABA (Fig. 1B) and ABA-PA (Fig. 1C). Remarkably, the intensity of the most abundant transition increased from 120 to 92,461 counts after ABA conjugation with PA.

For the optimization of chromatographic conditions, a C18 column typically used for the retention of low polar compounds was chosen. The use of methanol as organic solvent and the addition of low amounts of both formic acid and ammonium formate to the mobile phases were selected in order to promote the protonation in ESI+ [20]. Using a generic gradient from $5 \%$ to $95 \%$ of organic solvent, it could be deduced that ABA-PA eluted with c.a. $50 \%$ of organic solvent. In order to improve the chromatographic resolution, a slower gradient between $30 \%$ and $70 \%$ of organic solvent was finally selected.

During the development of the method, the presence of $A B A$ was identified in the plant-derived hemolytic agent saponin, which was initially used for the preparation of RBC extracts employed later for the method validation. The unequivocal presence of $A B A$ in saponin-containing solutions was determined by monitoring three different ion transitions of ABA-PA (Fig. S2). To avoid potential contaminations by plant ABA, an alternative saponin-free protocol based on acetone as organic solvent was established and used in this work for the extraction of RBCs.

\subsection{Method validation: $A B A$ extraction recovery and $R B C$ matrix effects}

Both SPE and LLE were studied as clean-up and pre-concentration steps. Obtained recoveries were $36 \pm 18 \%$ and $86 \pm 13 \%$ for SPE and LLE respectively. The 
relatively large amount of organic solvent in the extract might increase the breakthrough of the analyte being behind the low extraction recoveries obtained for SPE. A satisfactory linearity was achieved by the method with a correlation coefficient of $r=0.998$ for the studied range (0.05-10 ng ABA-PA mL $\left.{ }^{-1}\right)$. Because electrospray ionization is particularly prone to matrix effects, a specific study concerning this parameter was conducted by comparing the signals of a derivatized standard with and without the presence of the matrix $(n=6)$. The results proved that the multiple compounds present in a complex sample such as RBC extracts, in this case do not produce significant ionization enhancement or suppression, neither for ABA (89 $\pm 11 \%)$ nor for its ISTD $(95 \pm 12 \%)$. The chromatograms corresponding to the analysis of a blank RBC sample extract $\left(1.2 \times 10^{10}\right.$ cells $\left.\mathrm{mL}^{-1}\right)$ either alone or spiked with $0.15 \mathrm{ng} / \mathrm{mL}$ of $A B A$ are presented in Fig. 2.

As result of the ionization improvement provided by the PA-derivative formed, the limits of detection and quantification were respectively reduced to 0.03 and $0.15 \mathrm{ng}$ ABA $\mathrm{mL}^{-1}$, using a RBC-based matrix of $1.2 \times 10^{10}$ extracted cells $\mathrm{mL}^{-1}$. Up to date, these results validate this method as the most sensitive for ABA detection and quantification, despite using as matrix a very large amount of RBC extract.

Furthermore, intra-day and inter-day accuracy at both tested concentrations (0.15 and

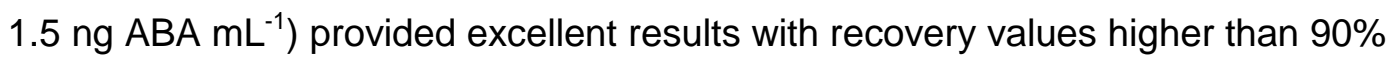
(Table S1). Finally, the validation results also showed the excellence of the method in terms of precision, with relative standard deviation values $\leq 10 \%$ in all cases. Because of the high ABA extraction recovery yield obtained, LLE was selected as the method of choice for $A B A$ detection and quantification in $P$. falciparum cultures.

3.3. Analysis of the possible effect of $C_{40}-C B P$ inhibitors on $A B A$ synthesis in $P$. falciparum cultures

The effect on the malaria parasite growth of two compounds known to interfere with the $\mathrm{C}_{40}-\mathrm{CBP}$, and therefore directly affecting the production of downstream carotenoids as precursor molecules for a potential ABA synthesis, was analyzed in $P$. falciparum in vitro cultures. Both fluridone and NDGA displayed activity against $P$. falciparum intraerythrocytic growth, with respective 50\% growth inhibitory concentrations (IC50) of 112.2 and $46.8 \mu \mathrm{M}$ (Figs. S3 and S4). The validated PAbased, LC-ESI-MS/MS method for ABA detection and quantification was applied to non-infected RBCs and to $P$. falciparum cultures in the trophozoite/schizont maturation stages. Parasitized RBC ( $p R B C$ ) cultures collected at $t_{24 h}$ from the untreated control 
sample in growth inhibition assays were extracted with the lyophilization/acetone method (see Supplementary material), subsequently purified by LLE plus PA derivatization and finally analyzed by LC-ESI-MS/MS (ISTD retention time of about 3.3$3.5 \mathrm{~min}$ and $355 \rightarrow 229 \mathrm{~m} / \mathrm{z}$ as major ABA-PA ion transition). No peak was detected matching the aforementioned conditions (Fig. S5), indicating either the absence of ABA in pRBC extracts or its presence in amounts below the LOD of $0.03 \mathrm{ng} \mathrm{mL}^{-1}$.

In pRBC extracts from growth inhibition assays after $24 \mathrm{~h}$ of incubation in the presence of fluridone and NDGA at their previously determined IC50s, an unknown compound was detected having the same molecular mass as ABA-PA and eluting from the liquid chromatography column at the ABA-d6-PA ISTD characteristic retention time ( 3.3 to $3.5 \mathrm{~min}$ ). Although this molecule displayed only the $355 \rightarrow 109 \mathrm{~m} / \mathrm{z}$ ion transition among the previously determined ABA-PA specific $355 \rightarrow$ 229/135/109 m/z major ion transitions (Table 1), it exhibited the predicted ABA behavior after treatment with $\mathrm{C}_{40^{-}}$ CBP inhibitors, presenting its maximum level in untreated $P$. falciparum cultures but being significantly reduced in the presence of fluridone and NDGA at their respective IC50s (Fig. 3).

\section{Discussion}

Several analytical approaches previously used for the quantification of ABA include ultraviolet or MS detection, either combined with high performance liquid chromatography [21] or capillary electrophoresis [22]. Initial MS methods were based on gas chromatography coupled to mass spectrometry [5,23-25], with the highest reported sensitivity obtained by forming the pentafluorobenzyl ester of ABA and subsequently detecting this derivative in an electron capture negative ionization source (ion trap mass spectrometer [24], or a quadrupolar instrument [23]). However, this technique is not well suited for the analysis of non-volatile substances or temperaturesensitive compounds and is less sensitive than more recent methodologies currently used for the detection of plant-derived ABA such as LC-ESI-MS/MS. Following the latter approach and working in negative ion mode, excellent detection and quantification limits have been reported ranging from 0.3 to $4 \mathrm{ng} \mathrm{g}^{-1}$ dry weight [26-28].

A further improvement in the detection of compounds bearing carboxylic acids had been achieved by means of their conjugation with PA [19], allowing in this manner for a more sensitive detection through positive ion mode; however, this strategy has never been explored for the analysis of ABA. We have successfully combined in this work an efficient LC-ESI-MS/MS system with ABA-PA derivatization, a conjugation step 
enabling $A B A$ to be quantified through selected reaction monitoring in positive ionization mode and ultimately resulting in a highly sensitive method with extraordinarily low LOD and LOQ respective values of 0.03 and $0.15 \mathrm{ng} \mathrm{ABA} \mathrm{mL} \mathrm{m}^{-1}$, with the additional difficulty of employing a complex $1.2 \times 10^{10} \mathrm{RBC} \mathrm{mL}^{-1}$ extract as matrix. In the most sensitive determination to date using an acetone-based cell extraction and in which $A B A$ was quantified through the negative ionization mode [26], the respective LOD and LOQ were 0.2 and $0.6 \mathrm{ng} \mathrm{g}^{-1}$ dry mass, ca. 7- and 4-fold higher, respectively, than those reported here. Such improvement in ABA detection sensitivity might be crucial for the analysis of this hormone in organisms presenting carotenoid biosynthetic routes analogous to those found in plants, such as parasites of the phylum Apicomplexa including Plasmodium and Babesia.

ABA is commonly quantified in plant extracts where sample availability is not a limitation. However, its determination in minute amounts from complex biological samples such as within intraerythrocytic apicomplexan pathogens producing carotenoids but lacking most of the ABA-controlled functions found in plants, requires a detailed optimization of the ABA chromatographic and mass spectrometric parameters. After choosing the right conditions for ABA purification and detection, we applied a full validation protocol using human RBCs as matrix in order to better characterize the newly developed method. The results presented here show that our optimized methodology is adequate for its intended purpose in terms of accuracy, precision and linearity.

Some of the reasons that might account for the failure in detecting ABA in $P$. falciparum cultures include: (i) its presence in a concentration below the limit of detection; (ii) the synthesis of this hormone only at a precise time of parasite maturation, such as the moment prior to egress from the host RBC [5], and thereby remaining below detectable amounts during most of the parasite's intraerythrocytic cycle; and (iii) the simultaneous existence of ABA derivatives having different molecular masses or showing different chromatography retention times. The amphiphilic properties of the molecule reflected in its high octanol/water partition coefficient $P$ of 123.03 units [29] indicate that at physiological $\mathrm{pH} \geq 99.5 \%$ of $A B A$ molecules will be in deprotonated (charged) form (Fig. S6), possibly leading to a widespread distribution of $A B A$ throughout the parasitized cell and the extracellular environment. This might eventually result in too low ABA amounts in pRBCs, below the LC-ESI-MS/MS detection level reported here of $0.03 \mathrm{ng} \mathrm{mL}^{-1}$.

Further research is required in order to ascertain if the detected unidentified compound whose levels are affected by ABA biosynthesis inhibitors is actually a 
modified derivative of $A B A$ or an interfering unrelated compound generating the $A B A$ PA-like $355 \mathrm{~m} / \mathrm{z}$ precursor ion. The application of open metabolomic approaches would be of great interest in order to unveil the identity of such potential ABA analogs, whose existence could significantly contribute to understanding the $P$. falciparum biological processes affected by $\mathrm{C}_{40}$-CBP inhibitors, which would in turn be of paramount importance for the development of future new antimalarial therapies.

\section{Conclusion}

A highly sensitive LC-ESI-MS/MS approach has been developed for ABA detection, with respective LOD/LOQ values of $0.03 / 0.15 \mathrm{ng} \mathrm{mL}^{-1}$, which has been validated in RBC matrices and finally applied to late-form pRBC extracts. Nevertheless, the presence of $A B A$ in the human malaria parasite $P$. falciparum remains still unresolved, although inhibitors such as NDGA affecting enzymes directly involved in its biosynthesis have been shown to arrest Plasmodium intraerythrocytic growth. Some likely explanations for such dichotomy are the synthesis of ABA by the intracellular pathogen in undetectable amounts, its production only at specific time points of the parasite's life-cycle, or the presence of a chemical derivative with different molecular mass and physicochemical properties. In this regard, further research is mandatory in order to finally resolve if $P$. falciparum and other Apicomplexa besides $T$. gondii are capable of synthesizing ABA. Such discovery would provide novel parasite-specific target pathways for the design of new drugs and improved therapies against Plasmodium and Babesia. Finally, the optimized method for ABA detection presented here could be applied as well to the study of this hormone in T. gondii.

\section{Acknowledgements}

This work was supported by grants BIO2014-52872-R from the Ministerio de Economía y Competitividad (MINECO), Spain, which included FEDER funds, and 2014-SGR-938 from the Generalitat de Catalunya, Spain. A fellowship from the Subprograma de Formación de Personal Investigador, MINECO, Spain, is acknowledged by E.M. The Spanish Health National System is acknowledged for the contract of O.J.P. (MS10/00576). IBEC and ISGlobal are members of the CERCA Programme, Generalitat de Catalunya. 


\section{References}

[1] J.L.N. Barratt, J. Harkness, D. Marriott, J.T. Ellis, and D. Stark, Importance of nonenteric protozoan infections in immunocompromised people, Clin. Microbiol. Rev., 23 (2010) 795-836.

[2] J. Wiesner, A. Reichenberg, S. Heinrich, M. Schlitzer, and H. Jomaa, The plastidlike organelle of apicomplexan parasites as drug target, Curr. Pharm. Des., 14 (2008) 855-871.

[3] I. Coppens, Targeting lipid biosynthesis and salvage in apicomplexan parasites for improved chemotherapies, Nat. Rev. Microbiol., 11 (2013) 823-835.

[4] R. Tonhosolo, F.L. D'Alexandri, V.V. de Rosso, M.L. Gazarini, M.Y. Matsumura, V.J. Peres, E.F. Merino, J.M. Carlton, G. Wunderlich, A.Z. Mercadante, E.A. Kimura, and A.M. Katzin, Carotenoid biosynthesis in intraerythrocytic stages of Plasmodium falciparum, J. Biol. Chem., 284 (2009) 9974-9985.

[5] K. Nagamune, L.M. Hicks, B. Fux, F. Brossier, E.N. Chini, and L.D. Sibley, Abscisic acid controls calcium-dependent egress and development in Toxoplasma gondii, Nature, 451 (2008) 207-210.

[6] S.A. Paiva and R.M. Russell, Beta-carotene and other carotenoids as antioxidants, J. Am. Coll. Nutr., 18 (1999) 426-433.

[7] J.C. Gray, J.A. Sullivan, J.H. Wang, C.A. Jerome, and D. MacLean, Coordination of plastid and nuclear gene expression, Philos. Trans. R. Soc. Lond. B Biol. Sci., 358 (2003) 135-145.

[8] W.I. Gruszecki and K. Strzalka, Carotenoids as modulators of lipid membrane physical properties, Biochim. Biophys. Acta - Mol. Basis Dis., 1740 (2005) 108115.

[9] T. Oritani and H. Kiyota, Biosynthesis and metabolism of abscisic acid and related compounds, Nat. Prod. Rep., 20 (2003) 414-425. 
[10] E. Nambara and A. Marion-Poll, Abscisic acid biosynthesis and catabolism, Annu. Rev. Plant Biol., 56 (2005) 165-185.

[11] M. Enomoto, S. Kawazu, S. Kawai, W. Furuyama, T. Ikegami, J. Watanabe, and K. Mikoshiba, Blockage of spontaneous $\mathrm{Ca}^{2+}$ oscillation causes cell death in intraerythrocitic Plasmodium falciparum, PLoS ONE, 7 (2012) e39499.

[12] S.-Y. Han, N. Kitahata, K. Sekimata, T. Saito, M. Kobayashi, K. Nakashima, K. Yamaguchi-Shinozaki, K. Shinozaki, S. Yoshida, and T. Asami, A novel inhibitor of 9-cis-epoxycarotenoid dioxygenase in abscisic acid biosynthesis in higher plants, Plant Physiol., 135 (2004) 1574-1582.

[13] M. Almeida Trapp, G.D. De Souza, E. Rodrigues-Filho, W. Boland, and A. Mithöfer, Validated method for phytohormone quantification in plants, Front. Plant Sci., 5 (2014) 417.

[14] S. Hou, J. Zhu, M. Ding, and G. Lv, Simultaneous determination of gibberellic acid, indole-3-acetic acid and abscisic acid in wheat extracts by solid-phase extraction and liquid chromatography-electrospray tandem mass spectrometry, Talanta, 76 (2008) 798-802.

[15] Z. Ma, L. Ge, A.S.Y. Lee, J.W.H. Yong, S.N. Tan, and E.S. Ong, Simultaneous analysis of different classes of phytohormones in coconut (Cocos nucifera L.) water using high-performance liquid chromatography and liquid chromatographytandem mass spectrometry after solid-phase extraction, Anal. Chim. Acta, 610 (2008) 274-281.

[16] A. Durgbanshi, V. Arbona, O. Pozo, O. Miersch, J.V. Sancho, and A. GómezCadenas, Simultaneous determination of multiple phytohormones in plant extracts by liquid chromatography-electrospray tandem mass spectrometry, J. Agric. Food Chem., 53 (2005) 8437-8442.

[17] A. Gómez-Cadenas, O.J. Pozo, P. García-Augustín, and J.V. Sancho, Direct analysis of abscisic acid in crude plant extracts by liquid chromatographyelectrospray/tandem mass spectrometry, Phytochem. Anal., 13 (2002) 228-234.

[18] T. Higashi, T. Ichikawa, S. Inagaki, J.Z. Min, T. Fukushima, and T. Toyo'oka, Simple and practical derivatization procedure for enhanced detection of 
carboxylic acids in liquid chromatography-electrospray ionization-tandem mass spectrometry, J. Pharm. Biomed. Anal., 52 (2010) 809-818.

[19] X. Li and A.A. Franke, Improved LC-MS method for the determination of fatty acids in red blood cells by LC-Orbitrap MS, Anal. Chem., 83 (2011) 3192-3198.

[20] J. Marcos and O.J. Pozo, Current LC-MS methods and procedures applied to the identification of new steroid metabolites, J. Steroid Biochem. Mol. Biol., 162 (2016) 41-56.

[21] P.I. Dobrev, L. Havlícek, M. Vagner, J. Malbeck, and M. Kamínek, Purification and determination of plant hormones auxin and abscisic acid using solid phase extraction and two-dimensional high performance liquid chromatography, $\mathrm{J}$. Chromatogr. A, 1075 (2005) 159-166.

[22] B.F. Liu, X.H. Zhong, and Y.T. Lu, Analysis of plant hormones in tobacco flowers by micellar electrokinetic capillary chromatography coupled with on-line large volume sample stacking, J. Chromatogr. A, 945 (2002) 257-265.

[23] P.H. Duffield and A.G. Netting, Methods for the quantitation of abscisic acid and its precursors from plant tissues, Anal. Biochem., 289 (2001) 251-259.

[24] A.G. Netting and B.V. Milborrow, Methane chemical ionization mass spectrometry of the pentafluorobenzyl derivatives of abscisic acid, its metabolites and other plant growth regulators, Biol. Mass Spectrom., 17 (1988) 281-286.

[25] E.A. Schmelz, J. Engelberth, H.T. Alborn, P. O'Donnell, M. Sammons, H. Toshima, and J.H. Tumlinson, Simultaneous analysis of phytohormones, phytotoxins, and volatile organic compounds in plants, Proc. Natl. Acad. Sci. U. S. A., 100 (2003) 10552-10557.

[26] R. Zhou, T.M. Squires, S.J. Ambrose, S.R. Abrams, A.R. Ross, and A.J. Cutler, Rapid extraction of abscisic acid and its metabolites for liquid chromatographytandem mass spectrometry, J. Chromatogr. A, 1010 (2003) 75-85.

[27] C.M.S. Silva, G. Habermann, M.R.R. Marchi, and G.J. Zocolo, The role of matrix effects on the quantification of abscisic acid and its metabolites in the leaves of 
Bauhinia variegata L. using liquid chromatography combined with tandem mass spectrometry, Braz. J. Plant Physiol., 24 (2012) 223-232.

[28] M. López-Carbonell and O. Jáuregui, A rapid method for analysis of abscisic acid (ABA) in crude extracts of water stressed Arabidopsis thaliana plants by liquid chromatography-mass spectrometry in tandem mode, Plant Physiol. Biochem., 43 (2005) 407-411.

[29] ChemAxon, http://www.chemaxon.com, 2016. 

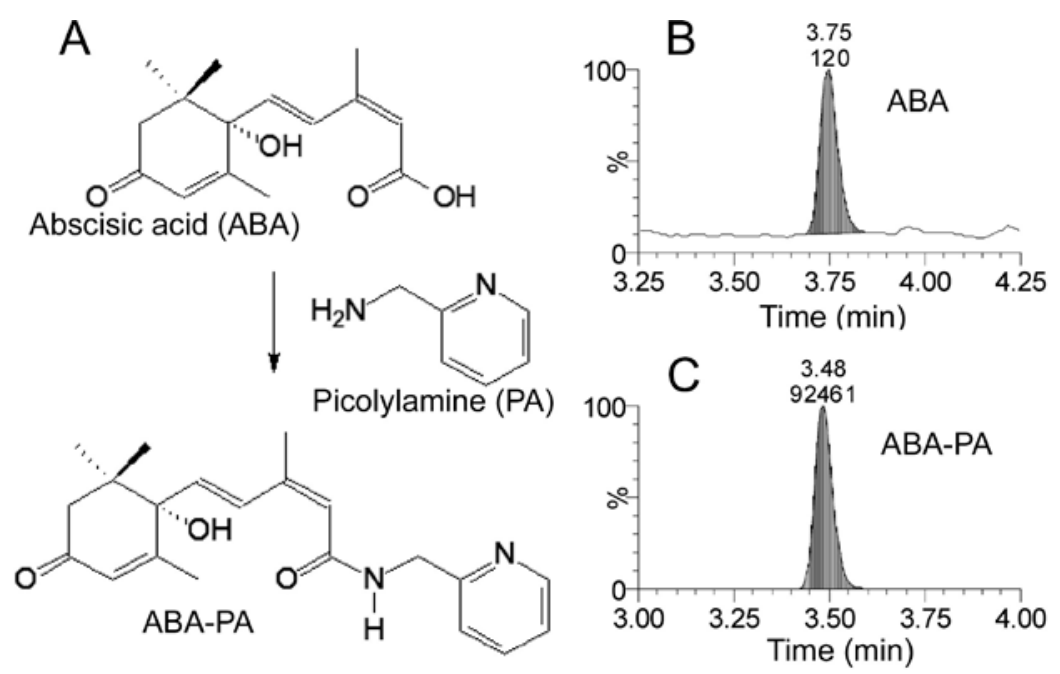

Fig. 1. $A B A$ derivatization with $P A$ and detection of $A B A-P A$ vs. underivatized $A B A$ by LC-ESI-MS/MS operating in SRM mode. (A) Derivatization reaction, (B) ABA detection in a solvent standard at $10 \mathrm{ng} \mathrm{mL}^{-1}$ using the ion transition $263 \rightarrow 153$ as optimized in [31] (retention time 3.75 min; peak integration 120 area units), and (C) ABA-PA detection in a solvent standard at $10 \mathrm{ng} \mathrm{mL}^{-1}$ using the $355 \rightarrow 229$ major ion transition (retention time 3.48 min; peak integration 92,461 area units).
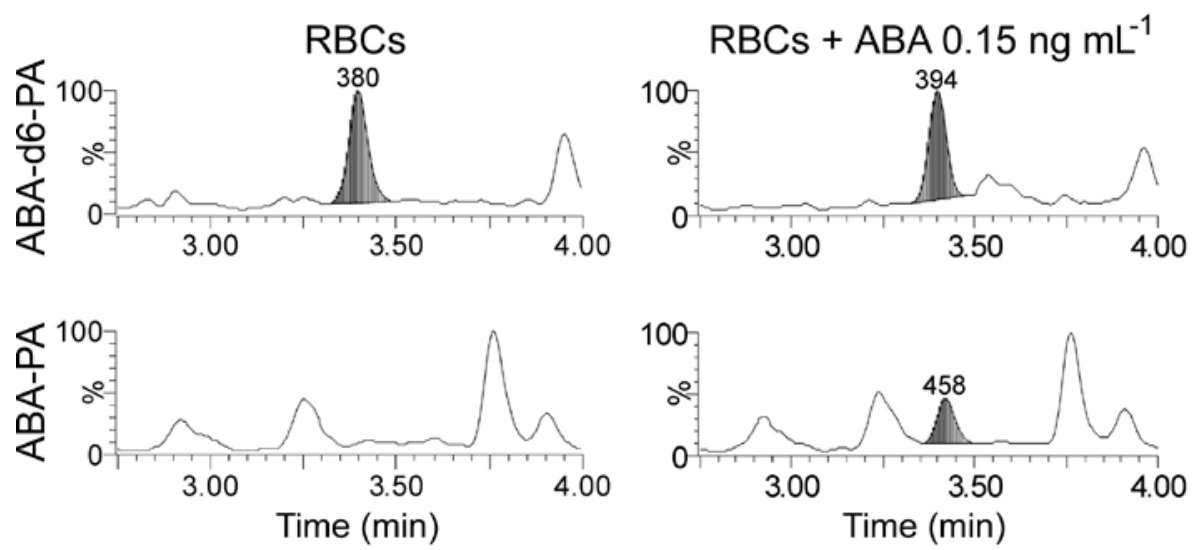

Fig. 2. LC-ESI-MS/MS chromatograms corresponding to ABA-PA and ABA-d6-PA (ISTD) for RBC blank samples $\left(1.2 \times 10^{10}\right.$ extracted cells $\left.\mathrm{mL}^{-1}\right)$ either alone or spiked with $0.15 \mathrm{ng} \mathrm{ABA} \mathrm{mL}^{-1}$. Peaks obtained at the characteristic ABA-PA $355 \rightarrow 229 \mathrm{~m} / \mathrm{z}$ and ISTD $361 \rightarrow 235 \mathrm{~m} / \mathrm{z}$ major ion transitions and detected at the specific retention time are shadowed. 

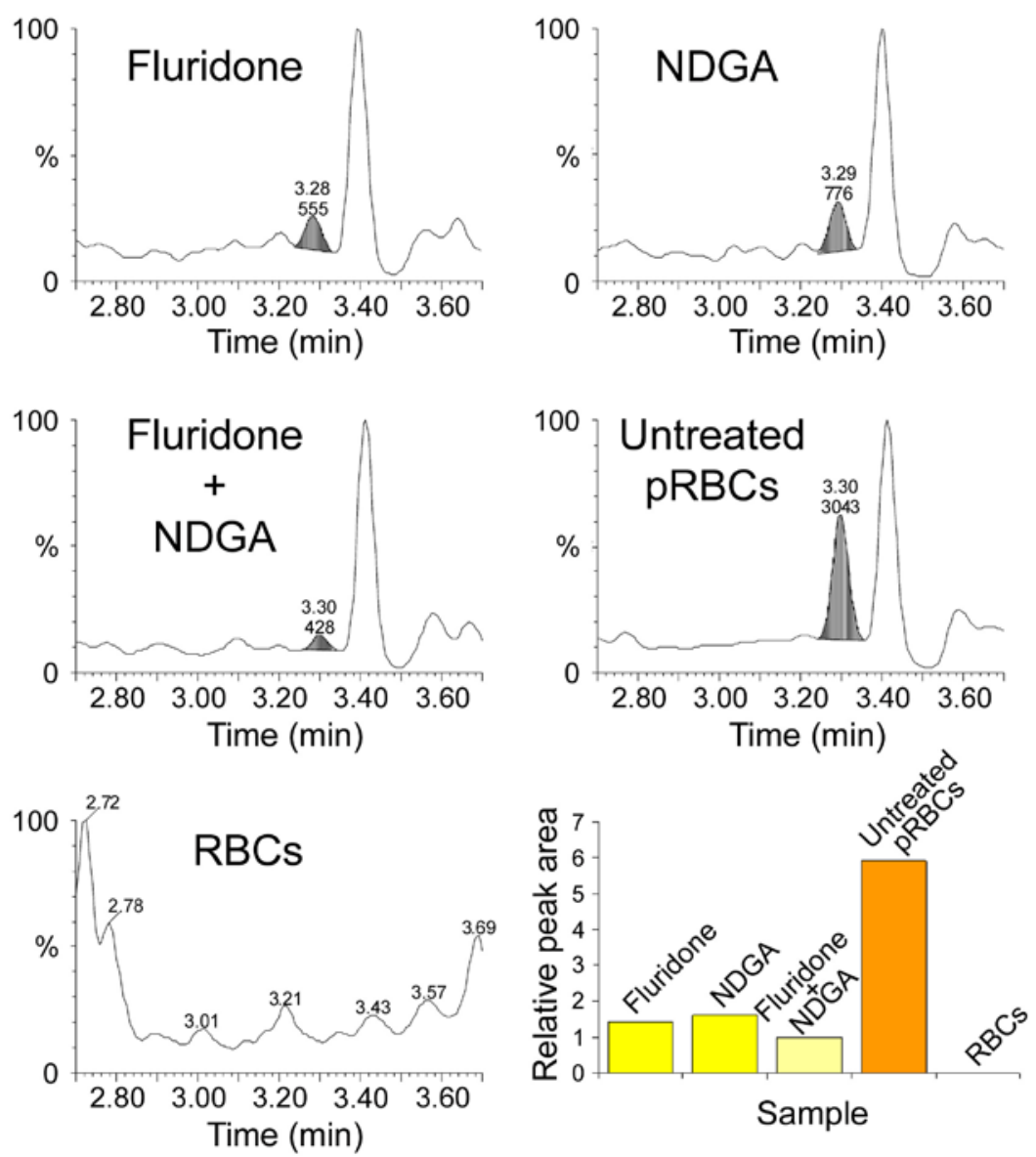

Fig. 3. Unknown compound detected in $P$. falciparum samples treated with $C_{40}-C B P$ inhibitors and exhibiting the hypothesized behavior of ABA. LC-ESI-MS/MS chromatograms corresponding to the total cell extracts obtained from cultures treated with $112 \mu \mathrm{M}$ fluridone, $47 \mu \mathrm{M}$ NDGA, and with both drugs combined the same respective concentrations, from untreated pRBCs, and from non-infected RBCs as negative control. The bottom right panel shows the corresponding areas under the shadowed peak detected at the ISTD retention time of $3.3 \mathrm{~min}$ and following the 355 $\rightarrow 109 \mathrm{~m} / \mathrm{z}$ ion transition. 


\title{
Supplementary material
}

\section{2-picolylamine derivatization for high sensitivity detection of abscisic acid in apicomplexan blood-infecting parasites}

\author{
Ernest Moles $^{\mathrm{a}, \mathrm{b}, \mathrm{c}, \star}$, Josep Marcos ${ }^{\mathrm{d}, \mathrm{e}}$, Santiago Imperial ${ }^{\mathrm{c}, \mathrm{f}}$, Oscar J. Pozo ${ }^{\mathrm{d}}$, and \\ Xavier Fernàndez-Busquets $\mathrm{s}^{\mathrm{a}, \mathrm{b}, \mathrm{c}, *}$ \\ ${ }^{a}$ Nanomalaria Group, Institute for Bioengineering of Catalonia (IBEC) \\ Baldiri Reixac 10-12, ES-08028 Barcelona, Spain \\ ${ }^{b}$ Nanomalaria Group, Barcelona Institute for Global Health (ISGlobal) \\ Rosselló 149-153, ES-08036 Barcelona, Spain \\ ${ }^{c}$ Nanoscience and Nanotechnology Institute (IN2UB), University of Barcelona \\ Martí i Franquès 1, ES-08028 Barcelona, Spain \\ ${ }^{d}$ Bioanalysis Research Group, IMIM, Hospital del Mar \\ Doctor Aiguader 88, ES-08003 Barcelona, Spain \\ ${ }^{e}$ Department of Experimental and Health Sciences, Universitat Pompeu Fabra \\ Doctor Aiguader 88, ES-08003 Barcelona, Spain \\ ${ }^{f}$ Department of Biochemistry and Molecular Biomedicine, University of Barcelona \\ Avda. Diagonal 643, ES-08028 Barcelona, Spain
}

* Corresponding author at:

Nanomalaria Group, ISGlobal, Rosselló 149-153, ES-08036, Barcelona, Spain.

E-mail address: ernest.moles@isglobal.org; xfernandez_busquets@ub.edu 


\section{Supplementary methods}

Materials

Except where otherwise indicated, reagents were purchased from Merck \& Co., Inc. (Kenilworth, NJ, USA), and reactions were performed at room temperature (22 to $\left.24^{\circ} \mathrm{C}\right)$.

Plasmodium falciparum in vitro culture and growth inhibition assays

The 3D7 $P$. falciparum strain was grown in vitro in group $B$ human erythrocytes using previously described conditions [1]. Parasites (thawed from glycerol stocks) were cultured at $37{ }^{\circ} \mathrm{C}$ in Petri dishes with red blood cells (RBCs) at $3 \%$ hematocrit in Roswell Park Memorial Institute (RPMI) complete medium containing Albumax II (RPMI-A, Invitrogen), supplemented with $2 \mathrm{mM} \mathrm{L-glutamine} \mathrm{and} \mathrm{under} \mathrm{a} \mathrm{gas} \mathrm{mixture} \mathrm{of}$ $92 \% \mathrm{~N}_{2}, 5 \% \mathrm{CO}_{2}$, and $3 \% \mathrm{O}_{2}$. For culture maintenance, parasitemia was kept below $5 \%$ late forms and $10 \%$ early forms by dilution with freshly washed RBCs and the medium was changed every 1-2 days. Synchronized cultures in early ring stages (0-24 h post-invasion) were obtained by $5 \%$ sorbitol lysis [2]. Late-form trophozoite and schizont stages (24-36 $\mathrm{h}$ and $36-48 \mathrm{~h}$ post-invasion, respectively) were purified in $70 \%$ Percoll (GE Healthcare) [2,3]. Parasitemia was determined by microscopic counting of blood smears fixed briefly with methanol and stained for 10 min with Giemsa (Merck Chemicals) diluted 1:10 in Sorenson's buffer, $\mathrm{pH}$ 7.2. Slides were observed with a Nikon Eclipse 50i (Japan) optical microscope and pictures were taken with a Nikon Digital Sight DS-U2 camera.

For $P$. falciparum in vitro growth inhibition assays, cultures synchronized (>95\%) in the early ring maturation stage were brought to $6 \%$ hematocrit and $1 \%$ parasitemia by dilution with fresh RBCs. After adding one culture volume of $2 \times$ concentrated drug solution in RPMI-A to achieve 3\% hematocrit, cultures were seeded in 96-well plates (Merck Chemicals) and incubated for a complete $48 \mathrm{~h}$ growth cycle under the conditions described above. For growth inhibition determination, samples were processed and analyzed by flow cytometry as described elsewhere [4]. Briefly, cultures were diluted 1:100 in $1 \times$ phosphate buffered saline (PBS) and the nuclei of $P$. falciparum-infected RBCs (pRBCs, the only nucleated cells present in the culture) were stained by addition of $0.1 \mu \mathrm{M}$ Syto11 (Thermo Fisher Scientific, Inc.) in the final mixture before proceeding to their analysis by a BD LSRFortessa flow cytometer (Becton, Dickinson and Company, NJ, USA). 
$P$. falciparum cultures at 6\% parasitemia and 3\% hematocrit synchronized in ring stages were washed several times with RPMI-A and cultured in the presence of $\mathrm{C}_{40^{-}}$ carotenoid biosynthetic pathway $\left(\mathrm{C}_{40}-\mathrm{CBP}\right)$ inhibitors for $24 \mathrm{~h}$ until appearance of pRBCs at the trophozoite and schizont late forms. Fluridone and nordihydroguaiaretic acid (NDGA) were assayed at their respective previously determined in vitro IC50 (112.2 $\mu \mathrm{M}$ and $46.8 \mu \mathrm{M})$, separately and in combination. The same volume of methanol, used as solvent for both inhibitors, was tested in parallel as control $(0.1 \% \mathrm{v} / \mathrm{v}$ methanol/culture). An additional control containing only uninfected RBCs at $3 \%$ hematocrit was also included. After the $24 \mathrm{~h}$ incubation period in the presence of $\mathrm{C}_{40^{-}}$ CBP inhibitors, $P$. falciparum cultures were spun down and the cell pellets were extracted through the lyophilization/acetone method (see below) as an adaptation of already established protocols for ABA purification [5-7]. In parallel to cell extraction, small aliquots of cultures in trophozoite stage were diluted 7.5-fold in RPMI, supplemented with new RBCs to obtain 3\% hematocrit, and further incubated for an additional $24 \mathrm{~h}$ in order to determine the parasite reinvasion capacity after treatment.

\section{Lyophilization/acetone cell extraction method}

Pelleted cells $\left(1.2 \times 10^{10}\right.$ total cells) contained throughout the whole extraction process in freezing-suitable polypropylene tubes were directly frozen in dry ice/ethanol and lyophilized overnight. The resulting pellets were thereafter weighted and solubilized in 8 volumes of ice-cold extraction solution (80:19:1 acetone:water:acetic acid). Samples were homogenized by vortex, incubated for $15 \mathrm{~min}$ and spun down $\left(10,000 \mathrm{~g}, 10 \mathrm{~min}, 4{ }^{\circ} \mathrm{C}\right)$. The supernatant obtained was stored at $4{ }^{\circ} \mathrm{C}$ and the remaining pellet was solubilized again in 8 volumes of ice-cold extraction solution, repeating the procedure two more times. All three supernatants were subsequently pooled and dried, first under $\mathrm{N}_{2}$ flow and finally lyophilized for a complete removal of solvent. 


\section{Supplementary results}

Effect of inhibitors of the $C_{40}-C B P$ on $P$. falciparum growth

The effect on the malaria parasite growth of two compounds known to interfere

with the $\mathrm{C}_{40}-\mathrm{CBP}$, and therefore directly affecting the production of downstream carotenoids as precursor molecules for a potential ABA synthesis, was analyzed in $P$. falciparum in vitro cultures. Both fluridone and NDGA displayed activity against $P$. falciparum intraerythrocytic growth, with respective $50 \%$ growth inhibitory concentrations (IC50) of 112.2 and $46.8 \mu \mathrm{M}$ (Figs. S3 and S4), in accordance with other $\mathrm{C}_{40}$-CBP inhibitors tested in $P$. falciparum and $T$. gondii $[5,8]$. In growth inhibition assays, fluridone- and NDGA-treated $P$. falciparum cultures displayed a significant developmental delay when compared to untreated controls (Fig. S4), as illustrated by an increase in the number of parasites exhibiting a picnotic nucleus (phenotype indicative of cell death) after $24 \mathrm{~h}$ incubation in the presence of either inhibitor, a feature which was exacerbated when parasites were treated simultaneously with both drugs. At the end of growth inhibition assays, $48 \mathrm{~h}$ after treatment started, a reduction of the number of parasites in ring early maturation forms could be observed; in addition, treated samples contained late stages prior to egression in significantly larger numbers than untreated control samples. These results strongly suggested a significant effect of fluridone and NDGA in impairing parasite development and replication. Because the observed $P$. falciparum growth-delayed phenotype was analogous to that of the ABA-producing $T$. gondii parasite, and considering the already known activity of fluridone and NDGA as inhibitors of ABA biosynthesis, we proceeded to the optimization of $A B A$ detection and the design of a validation method to be later applied to $P$. falciparum culture cell extracts. 


\section{Supplementary figures}

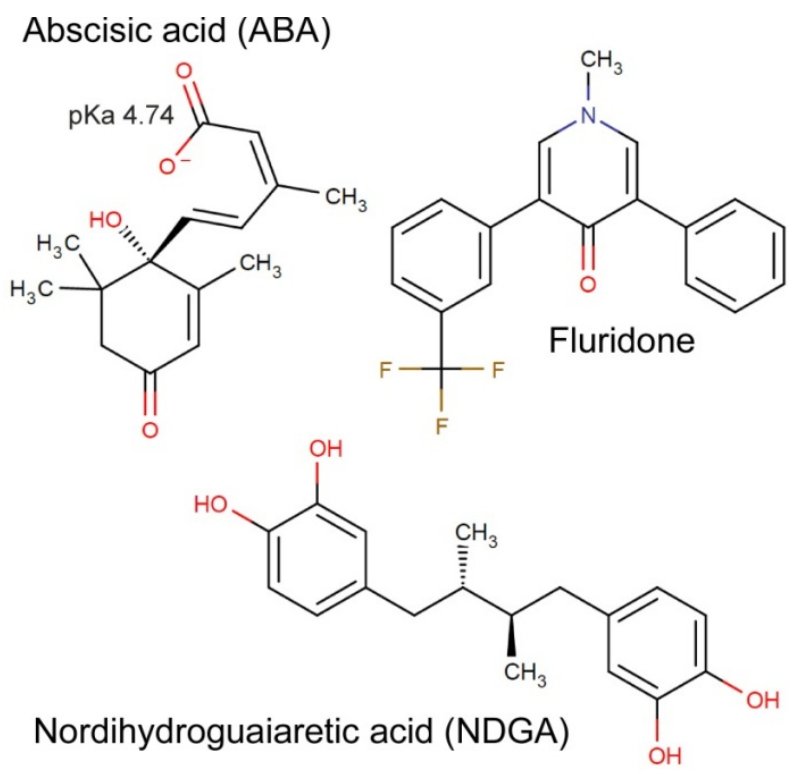

Fig. S1. Molecular structures of ABA, fluridone, and NDGA. From [9].
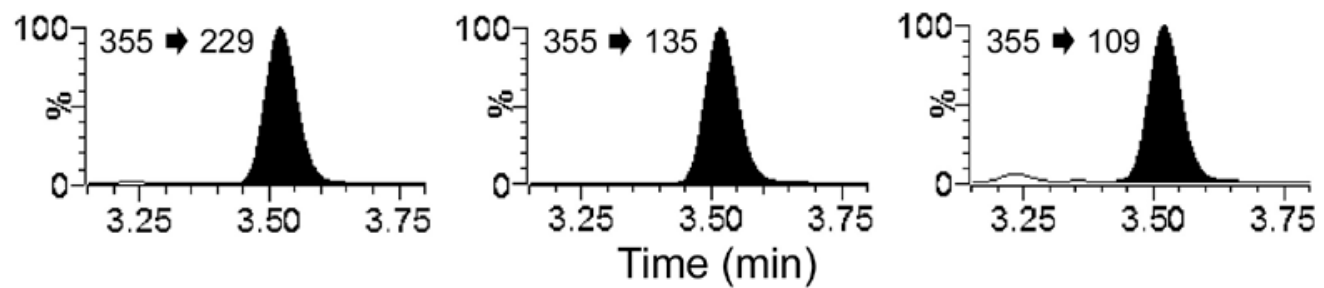

Fig. S2. Detection of $A B A$ in a $0.15 \%$ v/v saponin solution in PBS. LC-ESI-MS/MS chromatograms show the three major ion transitions $(\mathrm{m} / \mathrm{z})$ of ABA-PA.

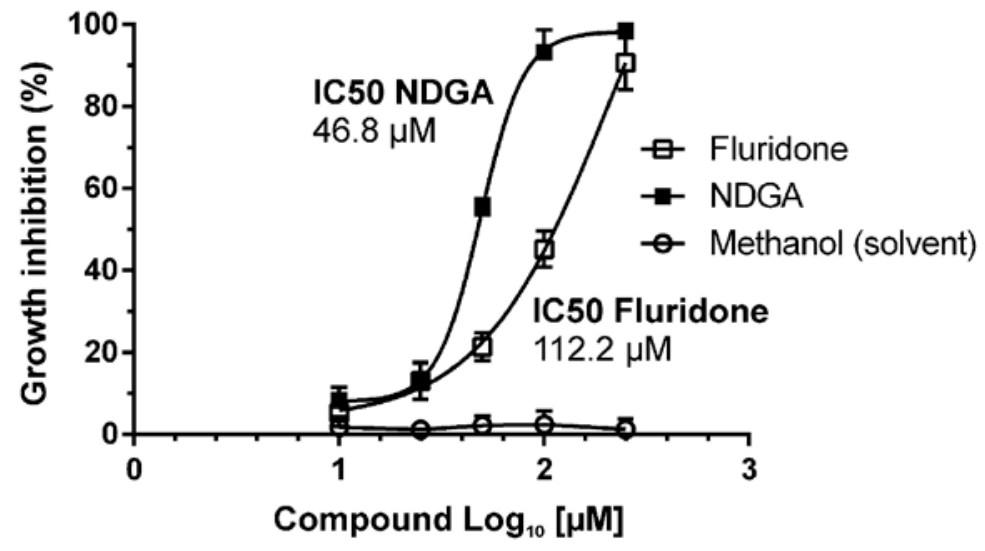

Fig. S3. P. falciparum growth inhibition assays of the effect of fluridone and NDGA added to ring stage cultures and analyzed after one replication cycle ( $48 \mathrm{~h}$ incubation). Bars indicate standard deviations. 


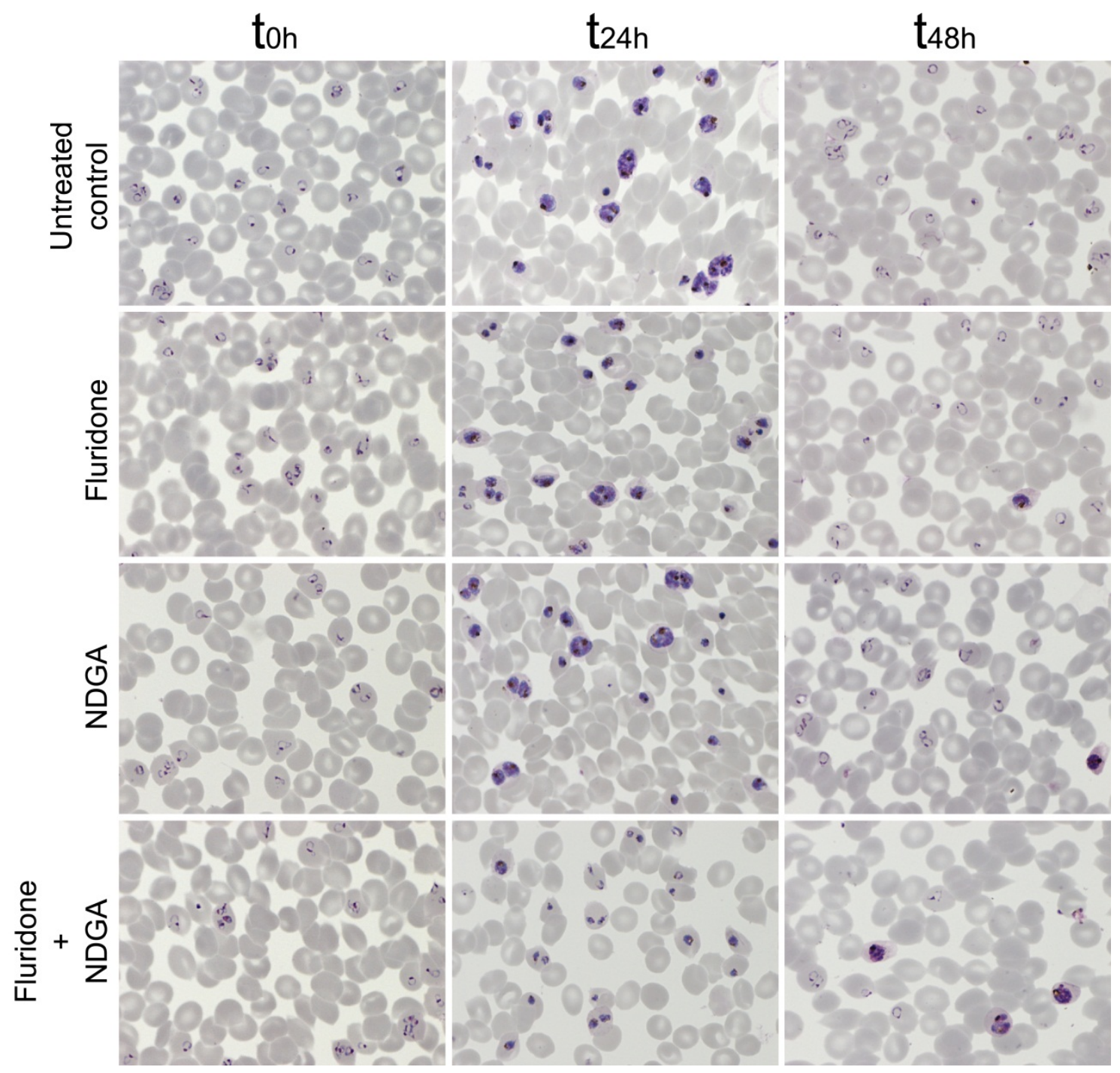

Fig. S4. Microscopic images of Giemsa-stained $P$. falciparum cultures corresponding to growth inhibition assays in the presence of fluridone and NDGA tested at their respective IC50s determined in the assay presented in Fig. S3. Images were obtained at $\mathrm{t}_{\mathrm{oh}}$ (parasites at ring stage), $\mathrm{t}_{24 \mathrm{~h}}$ (trophozoite and schizont stages), and $\mathrm{t}_{48 \mathrm{~h}}$ (ring stages again in untreated samples). Parasites collected at $t_{24 h}$ samples were employed later for ABA determination in P. falciparum cultures. 

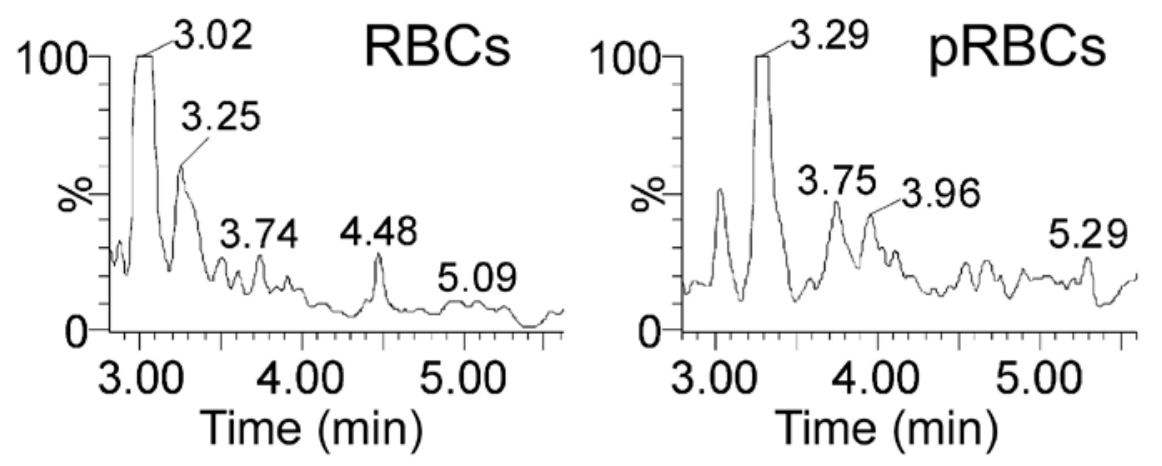

Fig. S5. LC-ESI-MS/MS chromatograms corresponding to ABA analysis in RBC and pRBC cell extracts obtained from $P$. falciparum untreated cultures at the trophozoite/schizont intraerythrocytic late stages, with no peak detected using the ABAPA specific parameter conditions.

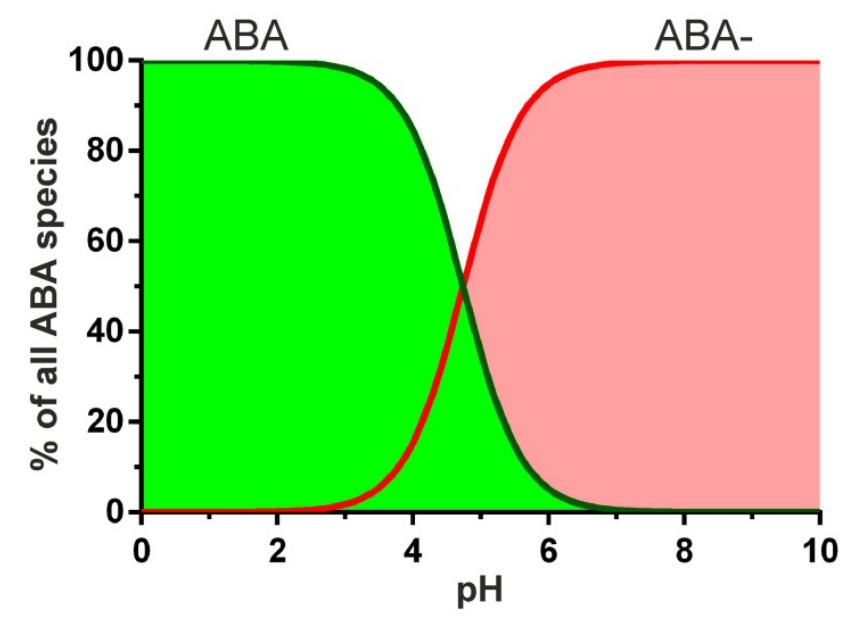

Fig. S6. ABA ionization species in solution as a function of $\mathrm{pH}$.

Table S1. Recovery analysis of intra- and inter-day assays at high- and low-level ABA concentrations.

\begin{tabular}{lcc}
\cline { 2 - 3 } & Intra-day $(\mathrm{n}=6)$ & Inter-day $(\mathrm{n}=12)$ \\
\hline Low level $\left(0.15 \mathrm{ng} \mathrm{ABA} \mathrm{mL}^{-1}\right)$ & $93 \pm 10 \%$ & $96 \pm 10 \%$ \\
\hline High level $\left(1.5 \mathrm{ng} \mathrm{ABA} \mathrm{mL}^{-1}\right)$ & $113 \pm 8 \%$ & $116 \pm 9 \%$ \\
\hline
\end{tabular}




\section{References}

[1] S.L. Cranmer, C. Magowan, J. Liang, R.L. Coppel, and B.M. Cooke, An alternative to serum for cultivation of Plasmodium falciparum in vitro, Trans. R. Soc. Trop. Med. Hyg., 91 (1997) 363-365.

[2] C. Lambros and J.P. Vanderberg, Synchronization of Plasmodium falciparum erythrocytic stages in culture, J. Parasitol., 65 (1979) 418-420.

[3] A. Radfar, D. Méndez, C. Moneriz, M. Linares, P. Marín-García, A. Puyet, A. Diez, and J.M. Bautista, Synchronous culture of Plasmodium falciparum at high parasitemia levels, Nat. Protoc., 4 (2009) 1899-1915.

[4] E. Moles, P. Urbán, M.B. Jiménez-Díaz, S. Viera-Morilla, I. Angulo-Barturen, M.A. Busquets, and X. Fernàndez-Busquets, Immunoliposome-mediated drug delivery to Plasmodium-infected and non-infected red blood cells as a dual therapeutic/prophylactic antimalarial strategy, J. Control. Release, 210 (2015) 217-229.

[5] K. Nagamune, L.M. Hicks, B. Fux, F. Brossier, E.N. Chini, and L.D. Sibley, Abscisic acid controls calcium-dependent egress and development in Toxoplasma gondii, Nature, 451 (2008) 207-210.

[6] L. Tian, D. DellaPenna, and J.A.D. Zeevaart, Effect of hydroxylated carotenoid deficiency on ABA accumulation in Arabidopsis, Physiol. Plant., 122 (2004) 314320.

[7] M. López-Carbonell and O. Jáuregui, A rapid method for analysis of abscisic acid (ABA) in crude extracts of water stressed Arabidopsis thaliana plants by liquid chromatography-mass spectrometry in tandem mode, Plant Physiol. Biochem., 43 (2005) 407-411.

[8] R. Tonhosolo, F.L. D'Alexandri, V.V. de Rosso, M.L. Gazarini, M.Y. Matsumura, V.J. Peres, E.F. Merino, J.M. Carlton, G. Wunderlich, A.Z. Mercadante, E.A. Kimura, and A.M. Katzin, Carotenoid biosynthesis in intraerythrocytic stages of Plasmodium falciparum, J. Biol. Chem., 284 (2009) 9974-9985.

[9] ChemAxon, http://www.chemaxon.com, 2016. 